\title{
Discount curve estimation by monotonizing McCulloch Splines
}

\author{
H.Dette, D.Ziggel \\ Ruhr-Universität Bochum \\ Fakultät für Mathematik \\ 44780 Bochum, Germany \\ E-mail: holger.dette@ruhr-uni-bochum.de \\ E-mail: daniel.ziggel@rub.de
}

August 2006

\begin{abstract}
In this paper a new method for monotone estimation of discount curves is proposed. The main idea of this approach is a simple modification of the commonly used (unconstrained) McCulloch Spline. We construct an integrated density estimate from the predicted values of the discount curve. It can be shown that this statistic is an estimate of the inverse of the discount function and the final estimate can be obtained by a numerical inversion. The resulting procedure is simple and we have implemented it in Excel and VBA, respectively. The performance is illustrated by two examples, in which the curve was previously estimated with an unconstrained McCulloch Spline.
\end{abstract}

\section{Introduction}

Yield curve estimation plays a central role in pricing fixed-income derivatives, risk management and for national central banks. Because the yield curve cannot be directly observed, and there are not enough zero coupon bonds existing, it has to be derived from observed market prices of coupon bearing bonds.

From the mathematical point of view it is equivalent to estimate the spot rates, the forward rates or the discount factors, where the discount factor $\delta(t)$ is the value of 1 unit money, which is paid in time $t$ [see for example Deacon, Derry (1994)]. In practice, one has different shape restrictions on these curves, which 
are based on economic theory. One very decisive condition is, that forward rates do not become negative. In other words, the discount curve has to be monotone decreasing. A second very crucial constraint is the initial condition $\delta(0)=1$.

Although there are a lot of different approaches and literature for estimating the yield curve existing [see Anderson et.al. (1996) for a review], there are only a few articles concerning the problem of nonnegative estimation of the forward rates ${ }^{1}$. Wets, Bianchi and Yang (2002) used, so-called, EpiCurves to obtain a monotone estimate of the discount curve. For this approach, which functional behavior is restricted to a subfamily of smooth curves, linear optimization with many constraints has to be used. Manzano and Blomvall (2004) presented a non-linear dynamic programming algorithm, which implements the forward rate positivity constraint for a one-parametric family of smoothness measures. Hagan and West (2005) introduced a monotone and convex spline [see also Kvasov (2000) for a textbook on shape restricted splines]. Here, the curve is guaranteed to be positive, if all the inputs are positive.

In this article we present an alternative procedure for the estimation of the discount curve, which can be used to monotonize any previously estimated (discount) curve. The method is extremely simple and uses an integrated density estimate from the predicted values of the unconstrained estimate of the discount function. It can be shown that this statistic is an estimate of the inverse of the discount function and the final (non-increasing) estimate can be obtained by numerical inversion. We will apply the new method to the widely used McCulloch Spline and illustrate its performance by two examples. The resulting procedure is very easy to handle and shows better results than ad hoc methods for monotonizing curves. A program implemented in Excel and VBA is available at $h t t p: / / w w w . r u h r-u n i-$ bochum.de/mathematik3/Ziggel.htm. We finally mention, that the proposed new procedure always yields nonnegative estimates of the forward rates and that it can be applied without any modification to monotonize other curve estimates.

\section{The McCulloch Spline}

Splines are very popular for estimating the term structure of interest rates. These functions are highly flexible and therefore suitable for most situations. A good review of the statistical aspects of this estimation method can be found in Eubank (1988).

McCulloch (1975) proposed a simple cubic spline model for the estimation of the discount curve, which shows a rather reliable performance in most circumstances. He suggested to choose $l-1$ knots, $k_{1}, \cdots, k_{l-1}$, with $k_{1}=0$ and $k_{l-1}$ corresponding to the longest maturity of a bond. For $j<l$ the $j$-th spline function is defined

\footnotetext{
${ }^{1}$ The following three are the only articles known to the authors.
} 
as

$$
\delta_{j}(t)= \begin{cases}0 & \text { if } t<k_{j-1} \\ \frac{\left(t-k_{j-1}\right)^{3}}{6\left(k_{j}-k_{j-1}\right)} & \text { if } k_{j-1} \leq t<k_{j} \\ \frac{\left(k_{j}-k_{j-1}\right)^{2}}{6}+\frac{\left(k_{j}-k_{j-1}\right)\left(t-k_{j}\right)}{2}+\frac{\left(t-k_{j}\right)^{2}}{2}-\frac{\left(t-k_{j}\right)^{3}}{6\left(k_{j+1}-k_{j}\right)} & \text { if } k_{j} \leq t<k_{j+1} \\ \left(k_{j+1}-k_{j-1}\right)\left[\frac{2 k_{j+1}-k_{j}-k_{j-1}}{6}+\frac{t-k_{j+1}}{2}\right] & \text { if } k_{j+1} \leq t,\end{cases}
$$

and for $j=l$ the function is given as:

$$
\delta_{l}(t)=t, \forall t
$$

The resulting spline estimator is finally obtained as the least squares fit of the function $\delta(t)=\sum_{j=1}^{l} \hat{\alpha}_{j} \delta_{j}(t)$ to the data.

From a practical point of view this method is not easy to handle, because splines are sometimes too flexible. Shea (1984) pointed out that spline functions, like any other numerical approximation technique, cannot yield reasonable estimates without the intelligent use of constraints. First, the user must choose the number and location of polynomial pieces, te that will serve as the building blocks of the spline model. The polynomial order and the degree of continuity of the spline function are also a matter of choice. If $n$ is the number of observed bonds, McCulloch (1975) suggested placing $l$ knots, where $l$ is the nearest integer to $\sqrt{n}$. Additionally, these knots have to be located, so that between two knots there are (nearly) the same number of bonds. There are more sophisticated rules for choosing smoothing parameters in spline estimation [see e.g. Eubank (1988)], but with this heuristic rule the McCulloch Spline is easy to implement and yields, under normal conditions, satisfactory results.

\section{-insert Table 1 about here-}

Unfortunately, the estimates obtained with the McCulloch Spline (and by other spline estimators) do not necessarily satisfy the shape restrictions from the economic theory. This is a general drawback of splines and is discussed in detail in two papers by Shea $(1984,1985)$. In particular it is possible, that the estimates of the forward rates become negative and we have illustrated this phenomenon in Figure 1 analyzing data from 81 Swiss mortgage bonds issued by the Pfandbriefbank of Swiss Cantonal Banks with settlement date 01.11.2003. The data is available at http://www.ruhr-uni-bochum.de/mathematik3/Ziggel.htm. Some summary statistics are listed in Table 1 . In Figure 1 we show the (unconstrained) estimates of the forward rates obtained with the McCulloch Spline, which are negative at some places. Nonnegative estimates of the forward rates can be avoided using parametric estimates for the discount curve. However, such methods are usually not very flexible. To illustrate this phenomenon, Figure 1 also shows 
the forward rates estimated by means of the techniques proposed by Nelson and Siegel (1987) and Svensson (1994). The parametric models suggested by these authors are often used by federal banks [see for example Schich (1996)]. The shapes of the parametric and spline curves are totally different. It is obvious that the parametric estimates are much smoother, but unfortunately the two methods are too inflexible and imprecise for many practical investigations.

-insert Figure 1 about here-

\section{Monotone smoothing by inversion}

In this section we briefly explain a very simple procedure for monotonizing curve estimates, which was recently proposed by Dette, Neumeyer and Pilz (2006). In the following we will use this method for monotonizing the McCulloch Spline described in Section 2, but it should be noted that this approach can be used to monotonize any unconstrained curve estimate. For this reason we describe it in the general nonparametric regression model

$$
Y_{i}=\delta\left(X_{i}\right)+\sigma\left(X_{i}\right) \epsilon_{i}, \quad i=1, \cdots, n,
$$

where $\left\{Y_{i}\right\}_{i=1}^{n}$ is a sample of observations [see e.g. Fan and Gijbels (1996)]. In our case this means, that $X_{i}$ is some time to maturity, $Y_{i}$ the corresponding discount factor and the function $\delta$ represents the discount curve. We assume that the explanatory variables $X_{i}$ vary in the interval $[0, T]$, with $T$ as the terminal node of the yield curve. We further assume that the random variables $\epsilon_{i}$ are i.i.d. with $E\left[\epsilon_{i}\right]=0, E\left[\epsilon_{i}^{2}\right]=1$. The regression function $\delta:[0, T] \rightarrow \mathbf{R}$ is assumed to be twice continuously differentiable. For the asymptotic analysis of the following estimate some further assumptions need to be made, but these will not be given here, because the focus of the present paper lies on applications [see Dette, Neumeyer and Pilz (2006) for technical details].

Our procedure starts with an unconstrained curve estimate, say $\hat{\delta}$. There are several proposals to estimate the function $\delta$ in model (3) [see e.g. Härdle (1990), Fan and Gijbels (1996) and Eubank (1988)] and in the following any of these estimates could be used as an initial (unconstrained) estimate for our method. In the applications discussed in the following section $\hat{\delta}$ will be the McCulloch Spline. If there is evidence that the regression function $\delta$ is (strictly) decreasing we define for a positive integer $N \in \mathbb{N}$

$$
\hat{\delta}_{i n v}(t):=\frac{T}{N h_{d}} \sum_{i=1}^{N} \int_{t}^{\infty} K_{d}\left(\frac{\hat{\delta}\left(T \frac{i}{N}\right)-u}{h_{d}}\right) d u,
$$


where $K_{d}$ denotes a positive and symmetric kernel with compact support, say $[-1,1]$, existing second moment and $h_{d}$ is a real (positive) parameter converging to 0 with increasing sample sizes, which is called bandwidth. Specific guidelines for the choice of $N$ and $h_{d}$ will be given in Section 4 . The statistic in (4) can be considered as an estimate of the inverse of the regression function $\delta$, which justifies the notation $\hat{\delta}_{i n v}$.

It can easily be motivated by replacing the points $T / N, 2 T / N, \ldots, T$ in (4) with an i.i.d. sample of uniformly distributed random variables, say $U_{1}, \ldots, U_{N} \sim$ $\mathcal{U}([0, T])$. If $\delta$ is a strictly decreasing function on the interval $[0, T]$ with a negative derivative, then

$$
\frac{1}{N h_{d}} \sum_{i=1}^{N} K_{d}\left(\frac{\delta\left(U_{i}\right)-u}{h_{d}}\right)
$$

is the classical kernel estimate of the density of the random variable $\delta\left(U_{1}\right)$ [see Silverman (1986)]. From elementary probability it follows that this density is given by

$$
-\frac{1}{T}\left(\delta^{-1}\right)^{\prime}(u) I_{[\delta(T), \delta(0)]}(u) .
$$

Consequently, if one integrates (5) appropriately and multiplies it by the factor $T$, one obtains an estimate of the function $\delta^{-1}$ at the point $t$. Finally, the unknown regression function $\delta$ is replaced by an appropriate estimate $\hat{\delta}$ and the random variables $U_{i}$ are substituted by the deterministic points $T i / N(i=1, \ldots, N)$, which yields the statistic $\hat{\delta}_{i n v}(t)$ defined in (4) as an estimate of the inverse $\delta^{-1}$ of the discount curve at the point $t$.

As the Kernel $K_{d}$ is positive with support $[-1,1]$, it follows that the estimate $\hat{\delta}_{i n v}$ is strictly decreasing, if, and only if, for all $i=1, \ldots, N$

$$
\min _{j \neq i}\left|\hat{\delta}\left(T \frac{i}{N}\right)-\hat{\delta}\left(T \frac{j}{N}\right)\right|<2 h_{d}
$$

Consequently, for fixed $h_{d}$ the estimate $\hat{\delta}_{i n v}$ is strictly decreasing if $N$ is chosen sufficiently large and throughout the present paper we assume that this is the case. Finally, a strictly decreasing estimate, say $\hat{\delta}_{\text {mon }}$, of the regression function $\delta$ is simply obtained by reflection of the function $\hat{\delta}_{i n v}$ at the line $y=x$ (here the index mon refers to the fact that the estimate is monotone decreasing). Note that the estimator $\hat{\delta}_{i n v}(t)$ is equal to 0 and 1 if

$$
t>\max _{i=1}^{N} \hat{\delta}\left(T \frac{i}{N}\right)+h_{d} \text { and } t<\min _{i=1}^{N} \hat{\delta}\left(T \frac{i}{N}\right)-h_{d}
$$

respectively. Because of this, the second crucial condition, $\hat{\delta}_{\text {mon }}(0)=1$, is trivially fulfilled, if $h_{d}$ is chosen sufficiently small and the preliminary unconstrained 
estimate satisfies $\hat{\delta}\left(T \frac{i}{N}\right) \leq 1$ for all $i$, which is normally the case in applications [see our examples in the following section].

For an increasing sample size and a bandwidth $h_{d}$ converging sufficiently fast to 0 it is shown in Dette, Neumeyer and Pilz (2006) that in cases, where the "true" regression function $\delta$ is in fact decreasing, the constrained estimate $\hat{\delta}_{\text {mon }}$ is first order asymptotically equivalent to the unconstrained estimate $\hat{\delta}$. Thus the new estimate $\hat{\delta}_{\text {mon }}$ is consistent and from a distributional point of view it shares the same properties as the unconstrained initial estimate $\hat{\delta}$ and is additionally strictly decreasing. In other words, whenever the distributional properties of the unconstrained estimate $\hat{\delta}$ are available, they can directly be transferred to the estimate $\hat{\delta}_{m o n}$. For example, if $\delta$ is twice continuously differentiable, monotone decreasing and a local linear estimate [see Fan and Gijbels (1996)] with kernel $K_{r}$ and bandwidth $h_{r}=o\left(n^{-2 / 5}\right)$ is used as initial unconstrained estimate, it is shown in Dette, Neumeyer and Pilz (2006) that $\hat{\delta}_{\text {mon }}$ is approximately normal distributed with mean

$$
\frac{h_{r}^{2} \delta^{\prime \prime}(t)}{2} \int K_{r}(u) u^{2} d u
$$

and variance

$$
\frac{\sigma^{2}(t)}{n h_{r} f(t)} \int K_{r}^{2}(u) d u
$$

where $\sigma^{2}$ is the variance function in model (3) and $f$ denotes the density of the explanatory variables.

Similarly, if the McCulloch spline is used as initial estimate $\hat{\delta}$, the constrained estimate $\hat{\delta}_{\text {mon }}$ is approximately normal distributed with mean 0 and variance

$$
u^{T}(t)\left(U^{T} U\right)^{-1} U^{T} \Sigma U\left(U^{T} U\right)^{-1} u(t)
$$

where $u(t)=\left(\delta_{1}(t), \ldots, \delta_{l}(t)\right)^{T}$ is the vector of spline functions and the matrices $\Sigma \in \mathbb{R}^{n \times n}$ and $U \in \mathbb{R}^{n \times l}$ are given by $\Sigma=\operatorname{diag}\left(\sigma^{2}\left(X_{1}\right), \ldots, \sigma^{2}\left(X_{n}\right)\right)$ and

$$
U=\left(\delta_{j}\left(X_{i}\right)\right)_{i=1, \ldots, n}^{j=1, \ldots, l} .
$$

\section{Implementation and computational issues}

In the present section, we will illustrate the monotonization method for the problem of constructing a decreasing estimate of the discount curve, where the McCulloch Spline is used as initial unconstrained estimate. We have implemented the monotonizing procedure in Excel and VBA, respectively.

For the application of the procedure we have used the McCulloch Spline, with knots placed as suggested in McCulloch (1975), for the preliminary estimate $\hat{\delta}$ in formula (4), which also requires the specification of a kernel $K_{d}$, a number $N$ of 
evaluation points and a (real valued) bandwidth $h_{d}$. For reasons of transparency we restrict ourselves to the Epanechnikov-kernel, but it is notable that other types of kernel estimators yield very similar results. The Epanechnikov-kernel is defined by

$$
K_{d}(u):=\left\{\begin{array}{cc}
\frac{3}{4}\left(1-u^{2}\right) & \text { if }|u|<1 \\
0 & \text { else }
\end{array}\right.
$$

easy to handle and fulfills the assumptions mentioned above. After integrating, equation (4) is a sum of $N$ terms multiplied by the constant $T /\left(N h_{d}\right)$, where for the $i$-th summand the integration yields 0 , if

$$
\hat{\delta}\left(T \frac{i}{N}\right)+h_{d}<t
$$

$h_{d}$, if

$$
\hat{\delta}\left(T \frac{i}{N}\right)-h_{d}>t
$$

and

$$
\frac{3}{4}\left[\hat{\delta}\left(T \frac{i}{N}\right)-t+\frac{2}{3} h_{d}-\frac{\left(\hat{\delta}\left(T \frac{i}{N}\right)-t\right)^{3}}{3 h_{d}^{2}}\right]
$$

else. It was observed by Dette, Neumeyer and Pilz (2006) empirically, that the choice of the bandwidth $h_{d}$ is not too critical as long as it is chosen sufficiently small. We use $h_{d}=0,001$ as bandwidth in estimate (4), while the parameter $N$ is chosen between 2300 and 2700 (but there will be no substantial differences if one uses $N=1000$ ).

The corresponding Excel and VBA-code for the calculation of the inverse $\hat{\delta}_{i n v}$ is very simple and available at http://www.ruhr-uni-bochum.de/mathematik3/Zig gel.htm. Note that this procedure requires the vector $(\hat{\delta}(T i / N))_{i=1, \ldots, N}$ of the unconstrained estimate and a set of points $\left(t_{1}, \cdots, t_{G}\right)$ as initial input.

The second step of the procedure consists in the inversion of the function $\hat{\delta}_{i n v}$. If the full curve has to be estimated and a set of points $\left(t_{i}, \hat{\delta}_{i n v}\left(t_{i}\right)\right)_{i=1}^{G}$ is available from the first step, this can easily be accomplished by plotting $\left(\hat{\delta}_{i n v}\left(t_{i}\right), t_{i}\right)_{i=1}^{G}$.

On the other hand, in a typical application a set of instruments is given and a set of discount factors at the maturity dates and/or cash flow dates has to be inferred, say $\hat{\delta}_{\text {mon }}(0) \geq \hat{\delta}_{\text {mon }}\left(x_{1}\right)=t_{1}>\ldots>\hat{\delta}_{\text {mon }}\left(x_{m}\right)=t_{m} \geq \hat{\delta}_{\text {mon }}(T)$. Note that the curve $\hat{\delta}_{i n v}:[0,1] \longrightarrow[0, T]$ is strictly decreasing, thus a simple bisection algorithm can be applied to calculate the function $\hat{\delta}_{m o n}$ at a given point.

More precisely, for the calculation of the value $t_{m}$ with $\hat{\delta}_{i n v}\left(t_{m}\right)=x_{m}$ we start with the interval $\left[\hat{\delta}_{i n v}(1), \hat{\delta}_{i n v}(0)\right]$ and determine $\hat{\delta}_{i n v}(0.5)$. If $\hat{\delta}_{i n v}(0.5) \leq x_{m}$, then $t_{m} \in\left[\hat{\delta}_{i n v}(0.5), \hat{\delta}_{i n v}(0)\right]$ otherwise $t_{m} \in\left[\hat{\delta}_{i n v}(1), \hat{\delta}_{i n v}(0.5)\right)$. We now repeat this procedure and obtain successively ranges of length

$$
\left(\hat{\delta}_{i n v}(0)-\hat{\delta}_{i n v}(1)\right) 2^{-\ell} ; \quad \ell=1,2, \ldots
$$


for the value $t_{m}$. This procedure converges very fast and only a few steps are required for a precise calculation of $t_{m}$. We now continue this procedure for the determination of the value $t_{j}$ with $\hat{\delta}_{i n v}\left(t_{j}\right)=x_{j}$ starting with the interval $\left[\hat{\delta}_{i n v}(1), \hat{\delta}_{i n v}\left(t_{j+1}\right)\right]$ instead of $\left[\hat{\delta}_{i n v}(1), \hat{\delta}_{i n v}(0)\right]$, where $j=m-1, \ldots, 1$.

\section{$5 \quad$ Empirical tests}

In this section we investigate the new estimate in two examples and compare it with several alternative proposals. As mentioned above the initial estimate is obtained from the classical McCulloch Spline, while the corresponding monotonizations are obtained from (4) and an inversion.

Our first example considers the situation investigated in Section 2. We have estimated the term structure of interest rates of 81 Swiss mortgage bonds issued by the Pfandbriefbank of Swiss Cantonal Banks with settlement date 01.11.2003. The classical estimation with the McCulloch spline yields highly fluctuating forward rates, which become even negative [see Figure 2]. The new estimate $\hat{\delta}_{\text {mon }}$, which was obtained with $N=2340$, modifies the initial estimate in regions where it is not decreasing. In Table 2 we show the knots of the McCulloch spline and the spot and forward rates at these knots obtained by the constrained and unconstrained estimate. We observe substantial differences between rates obtained by both methods, even in regions, where the initial unconstrained estimate is already decreasing. On the other hand the differences between the discount factors are less visible. In particular there is a maximal difference of 79 base points between the discount factors of the constrained and the unconstrained estimate [see Figure 3].

$$
\begin{aligned}
& \text {-insert Table } 2 \text { about here- } \\
& \text {-insert Figure } 2 \text { about here- } \\
& \text {-insert Figure } 3 \text { about here- }
\end{aligned}
$$

Figure 4 shows a partially constructed example. We took Finnish government bonds with settlement date 30.06.2005 and manipulated these data. As the sample size was only 12, changing the coupon of 1 bond from $5 \%$ to $20 \%$ was enough to get negative forward rates [see the lower panel in Figure 4]. The summary statistics for these data can be found in Table 3, while the data is available at http://www.ruhr-uni-bochum.de/mathematik3/Ziggel.htm. This example is not unrealistic from a practical point of view, as some errors in the data often occur in practice. We calculated the monotone estimate with $N=2627$ and observed a similar picture as for the Swiss bonds. There exist substantial differences in 
the rates obtained by unconstrained and constrained estimation. Additionally we also observed small differences in the discount factors.

\author{
-insert Table 3 about here- \\ -insert Table 4 about here- \\ -insert Figure 4 about here-
}

In both cases the estimated forward rates seem to be more reliable and less fluctuating after monotonization. In addition the two estimates of the discount curves show a similar behavior in regions, where the unconstrained estimate is already decreasing. Moreover the shapes of the curves are much closer to each other then to curves obtained by parametrical models.

It was pointed out by the referees, that it is also of some interest to compare the new procedure with some alternative methods proposed in the literature. There exist several proposals to estimate the yield curve [see for example Linton, Mammen, Nielsen and Tanggaard (2001) or Hagan and West (2005)]. A detailed comparison of various monotone estimates in a more general context by means of a simulation study can be found in Dette and Pilz (2006). For reasons of brevity we restrict ourselves to an empirical comparison of the new method with two procedures, which have been proposed recently in the economic literature for estimating discount curves. Linton et al. (2001) proposed local linear smoothing for the estimation of yield curves. This procedure does not yield a monotone estimate of the discount curve, but if it is combined with the monotone decreasing least squares estimate [see Barlow, Bartholomew, Bremner and Brunk (1972)], a decreasing estimate of the discount curves is obtained, which is called PAVAestimate in the statistical literature. The local linear estimate requires a (real) smoothing parameter (bandwidth) $h_{r}$, which has been chosen by least squares cross validation in our study. A detailed description of this procedure can be found in Dette and Pilz (2006).

The second estimate considered in our comparison is the monotone preserving cubic spline proposed in Hagan and West (2005). For this procedure, we used equally spaced knots with $k_{i}-k_{i-1}=0.5$ and the McCulloch Spline was used to determine the initial values, which were interpolated by the monotone preserving cubic spline [see Hagan and West (2005) for more details]. Note that this procedure yields only to a local monotone estimate of the curve [see Hagan and West (2005)], while the PAVA estimate and the method proposed in this article produce a decreasing curve over the full range.

In Figures 5 and 6 we show the estimates of the discount curves and forward rates obtained from these methods in comparison to the new estimate proposed 
in this paper for the Swiss mortgage bonds and the Finnish government bonds, respectively.

We obtain a very similar picture for both data sets. As pointed out in the previous paragraph, the monotone preserving spline results in a local monotone estimate and as a consequence this method yields negative estimates of the forward rates. Moreover, this method produces even more negative estimates of the forward rates than the McCulloch Spline. It is also notable that - in contrast to the two other estimates - the monotone preserving spline produces more fluctuating curve estimates. The PAVA-method yields a decreasing estimate, but in some regions the corresponding curve has a slope close to 0 . The reason for this phenomenon is that this method determines the best $L^{2}$-approximation of the the unconstrained curve by a decreasing curve [see Mammen, Marron, Turlach, Wand (2001)]. As a consequence, nonnegative estimates of the forward rates are obtained by the PAVA-estimates, but the rates are 0 in regions where the initial local linear estimate is in fact not decreasing. For these reasons the PAVA-estimate seems unreliable for estimating forward rates.

$$
\begin{aligned}
& \text {-insert Figure } 5 \text { about here- } \\
& \text {-insert Figure } 6 \text { about here- }
\end{aligned}
$$

\section{Conclusions}

We have presented a new procedure to monotonize any prior unconstrained estimate of the discount curve. The method is very easy to realize and a procedure in Excel and VBA has been implemented, which is available at $h t t p: / / w w w . r u h r-u n i-$ bochum.de/mathematik3/Ziggel.htm. We have illustrated the application of the new estimate by two examples and have compared the new procedure with two alternative estimates, which were proposed recently in the literature. These tests show some improvement with respect to the estimation of the forward rates. The new estimate has a similar behavior as the initial unconstrained estimate where this is already decreasing. The resulting statistic is a decreasing estimate over the full observation region and it does not yield regions where the forward rates are close to 0 . Because of these advantages and its simplicity we recommend this method for practical investigations.

Acknowledgements. The work of the first author was supported by the Deutsche Forschungsgemeinschaft (SFB 475, Komplexitätsreduktion in multivariaten Datenstrukturen). We are also grateful to Dr.S.Siemßen from the BayernLB for his helpful comments on an earlier version of this paper. We would also like to thank 
two unknown referees for their helpful comments, which led to a substantial improvement of an earlier version of this paper.

\section{References}

1 Anderson N., et.al. (1996) Estimating and interpreting the Yield Curve, Wiley, New York

2 Barlow, R.E., Bartholomew, D.J. , Bremner, J.M., Brunk, H.D. (1972). Statistical inference under order restrictions: The theory and application of isotonic regression. Wiley, New York.

3 Deacon M., Derry A. (1994) Estimating the Term Structure of Interest Rates, Bank of England Working Paper, London

4 Dette, H., Pilz, K.F. (2006). A comparative study of monotone nonparametric kernel estimates. Journal of Statistical Computation and Simulation, Vol. 76, 41-56.

5 Dette H., Neumeyer N., Pilz K.F. (2006) A simple nonparametric estimator of a strictly monotone regression function, Bernoulli 12, 469-490.

6 Eubank R.L. (1988) Spline smoothing and nonparametric regression, Marcel Dekker, New York

7 Fan J., Gijbels I. (1996) Local Polynomial Modeling and Its Applications, Chapman and Hall, London

8 Härdle W. (1990) Applied Nonparametric Regression, Cambridge University Press, Cambridge

9 Hagan P., West G. (2005) Interpolation Methods For Curve Construction, to appear in Applied Mathematical Finance, early 2006

10 Kvasov B.I. (2000) Methods of shape-preserving spline approximation, Singapore: World Scientific

11 Linton, 0., Mammen, E., Nielsen, J. Tanggaard, C. (2001). Estimating yield curves by kernel smoothing methods, Journal of Econometrics, 105, 185-223.

12 Mammen, E., Marron, J.S., Turlach, B.A., Wand, M.P. (2001). A general projection framework for constrained smoothing. Statist. Sci. 16, 232-248. 
13 Manzano J., Blomvall J. (2004) Positive forward rates in the maximum smoothness framework, Quantitative Finance 4, 221-232, Oxford

14 McCulloch J.H. (1975) The Tax-Adjusted Yield Curve, The Journal of Finance 30, 811-830, Berkeley

15 Nelson C.S., Siegel A.F. (1987) Parsimonious Modeling of Yield Curves, Journal of Business, 60, 4, 473-489, Chicago

16 Schich S.T. (1996) Alternative Spezifikation der deutschen Zinsstrukturkurve und ihr Informationsgehalt hinsichtlich der Inflation, Diskussionspapier 8/96, Deutsche Bundesbank, Frankfurt

17 Shea G.S. (1984) Pitfalls in Smoothing Interest Rate Term Structure Data: Equilibrium Models and Spline Approximations, Journal of Financial and Quantitative Analysis, Vol. 19, Nr. 3, Washington

18 Shea G.S. (1985) Interest Rate Term Structure Estimation with Exponential Splines: A Note, The Journal of Finance 40, 319-325, Berkeley

19 Silverman B.W. (1986) Density estimation for statistics and data analysis, Monographs on Statistics and Applied Probability, London - New York: Chapman and Hall

20 Svensson L.E.O. (1994) Estimating an Interpreting Forward Interest Rates: Sweden 1992-1994, NBER Working Paper Nr. 4871, Cambridge

21 Wets R.J-B., Bianchi S., Yang L. (2002) Serious Zero-Curves, EpiSolutions Inc. 


\begin{tabular}{|c|c|}
\hline Time to maturity (years) & Coupons (\%) \\
\hline min: 0.389 & min: 1.625 \\
\hline max: 11.664 & max: 7.125 \\
\hline mean: 3.816 & mean: 3.929 \\
\hline stand. dev.: 2.813 & stand. dev.: 1.105 \\
\hline
\end{tabular}

Table 1: Summary statistics for the Swiss mortgage bonds. The data is available at http://www.ruhr-uni-bochum.de/mathematik3/Ziggel.htm.

\begin{tabular}{|l|c|c|c|}
\hline Knots & Discount factors & Spot rates & Forward rates \\
\hline 1.039 & $0.984(0.986)$ & $1.553(1.285)$ & $1.549(1.089)$ \\
\hline 1.372 & $0.986(0.985)$ & $1.005(1.16)$ & $-0.772(0.896)$ \\
\hline 2.0 & $0.971(0.969)$ & $1.5(1.5)$ & $5.327(4.789)$ \\
\hline 2.7 & $0.939(0.95)$ & $2.332(1.951)$ & $0.994(1.793)$ \\
\hline 3.372 & $0.951(0.943)$ & $1.498(1.754)$ & $-1.002(1.025)$ \\
\hline 4.25 & $0.929(0.928)$ & $1.734(1.748)$ & $4.057(4.170)$ \\
\hline 6.047 & $0.878(0.876)$ & $2.161(2.162)$ & $3.226(3.114)$ \\
\hline 8.039 & $0.812(0.81)$ & $2.595(2.6)$ & $4.086(4.187)$ \\
\hline 11.664 & $0.711(0.712)$ & $2.923(2.964)$ & $3.521(3.494)$ \\
\hline
\end{tabular}

Table 2: Knots of the unconstrained and constrained (in brackets) estimate with its corresponding discount factors, spot rates and forward rates for the Swiss mortgage bonds.

\begin{tabular}{|c|c|}
\hline Time to maturity (years) & Coupons (\%) \\
\hline min: 0.156 & min: 2.75 \\
\hline max: 10.011 & max: 20 \\
\hline mean: 3.983 & mean: 6.148 \\
\hline stand. dev.: 2.993 & stand. dev.: 4.707 \\
\hline
\end{tabular}

Table 3: Summary statistics for the Finnish government bonds. The data is available at http://www.ruhr-uni-bochum.de/mathematik3/Ziggel.htm.

\begin{tabular}{|l|c|c|c|}
\hline Knots & Discount factors & Spot rates & Forward rates \\
\hline 2.011 & $0.896(0.894)$ & $5.45(5.753)$ & $5.758(5.911)$ \\
\hline 5.208 & $0.888(0.868)$ & $2.288(2.709)$ & $-0.311(0.648)$ \\
\hline 10.011 & $0.718(0.723)$ & $3.313(3.293)$ & $7.589(6.923)$ \\
\hline
\end{tabular}

Table 4: Knots of the unconstrained and constrained (in brackets) estimate with its corresponding discount factors, spot rates and forward rates for the Finnish government bonds. 


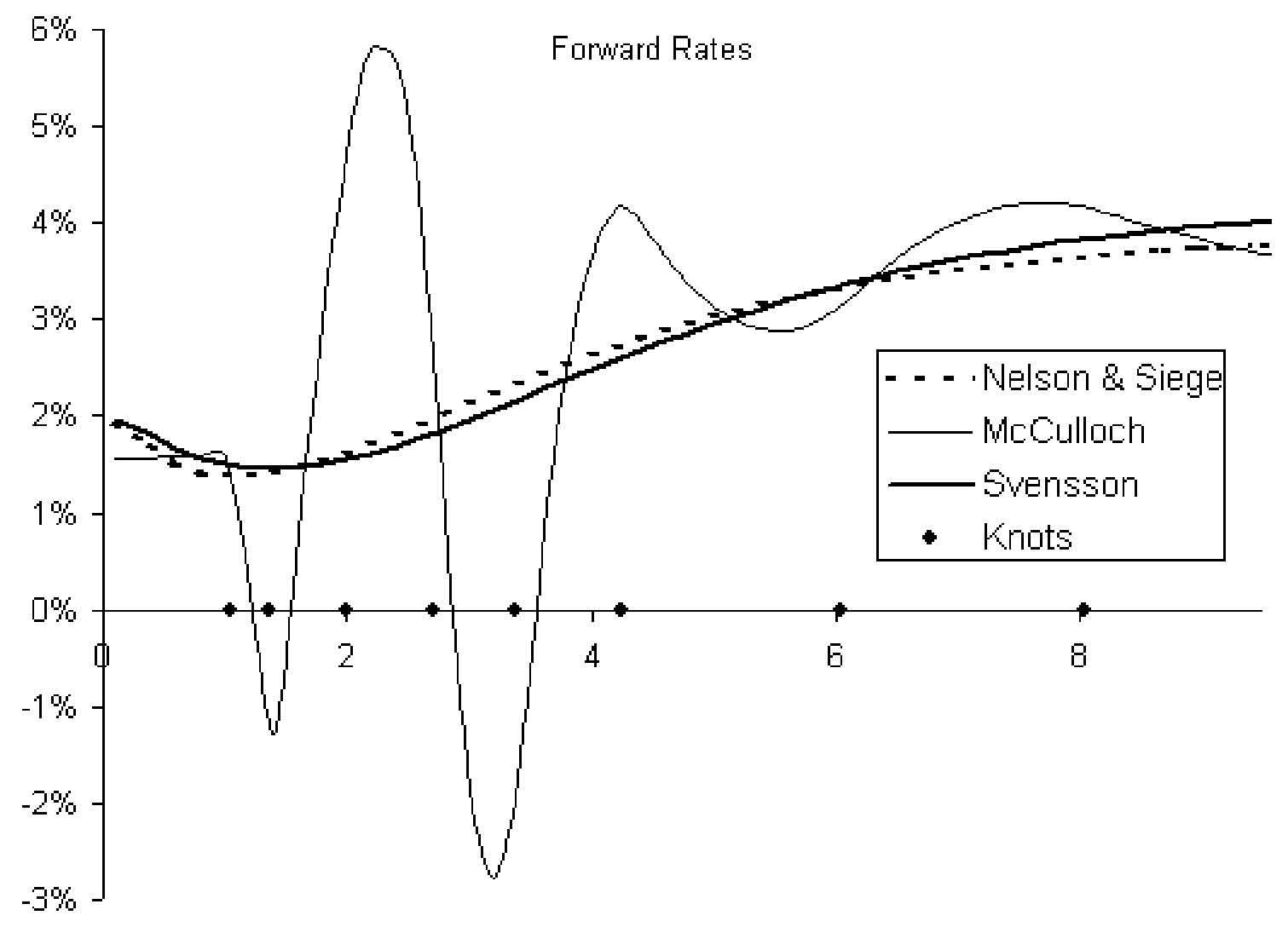

Figure 1: Estimated forward rates of Swiss mortgage bonds. 

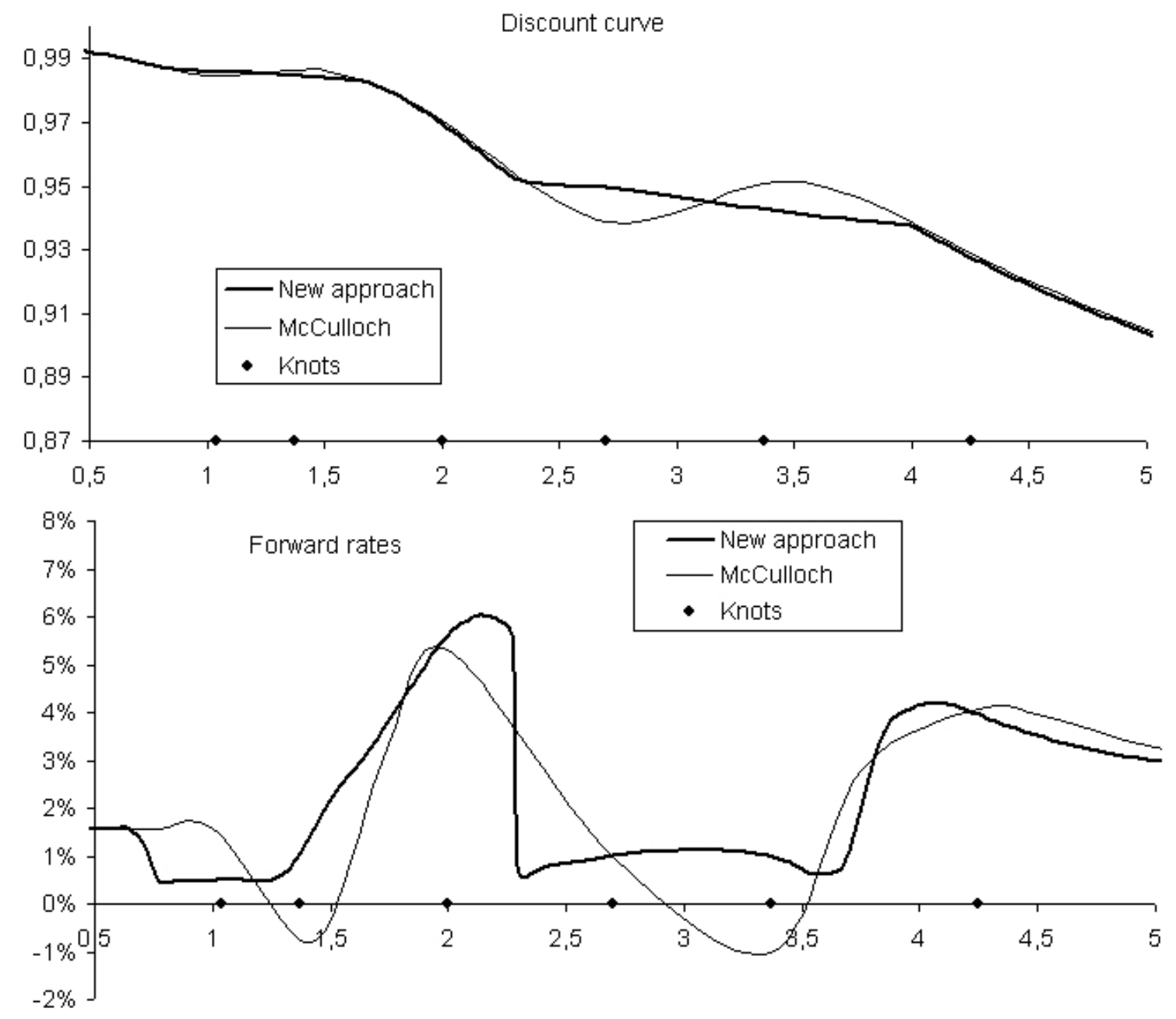

Figure 2: Estimated discount curve and forward rates of Swiss mortgage bonds. 


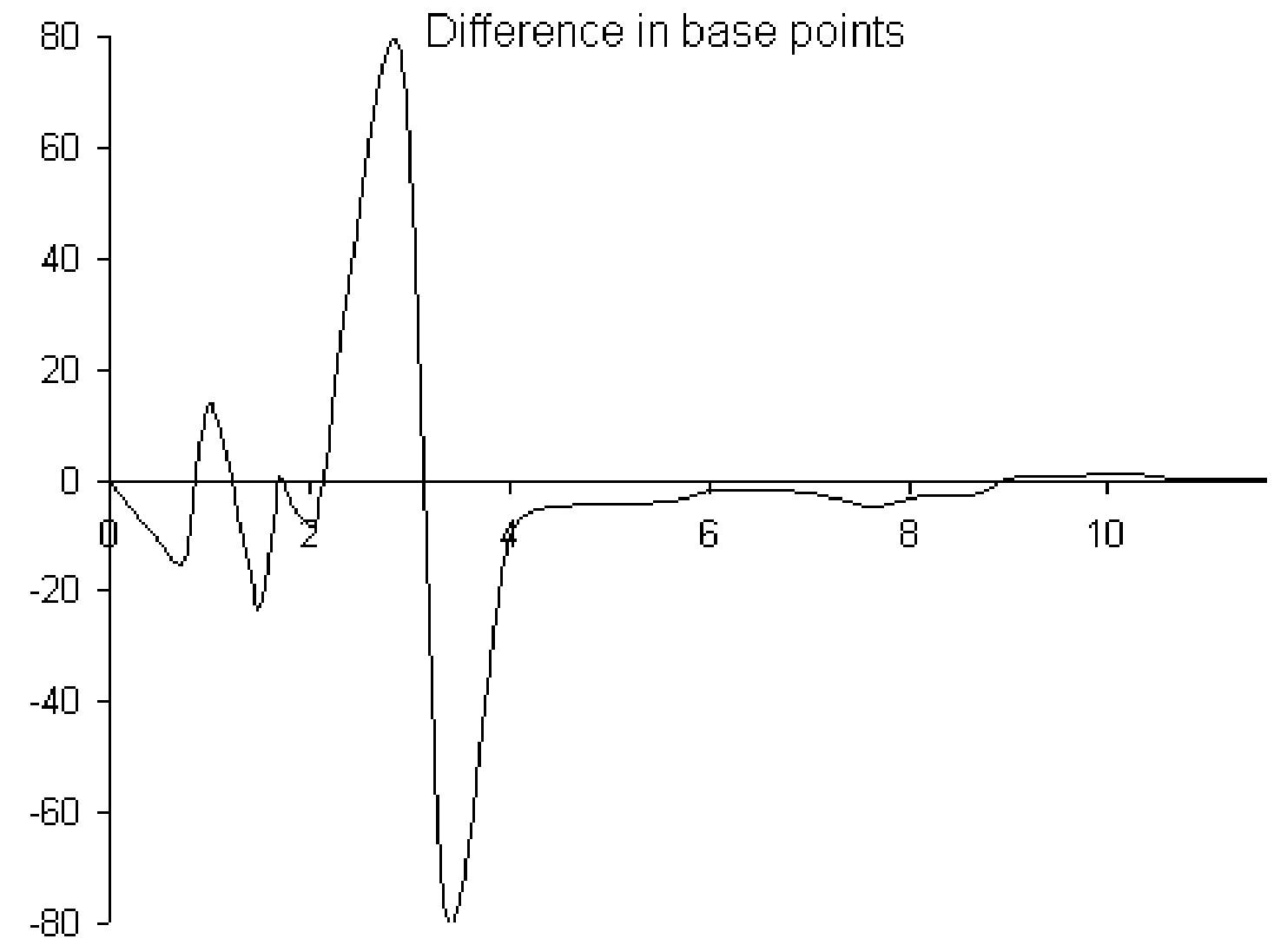

Figure 3: Difference in base points between discount factors of the initial estimate and its monotonization for the Swiss mortgage bonds. 

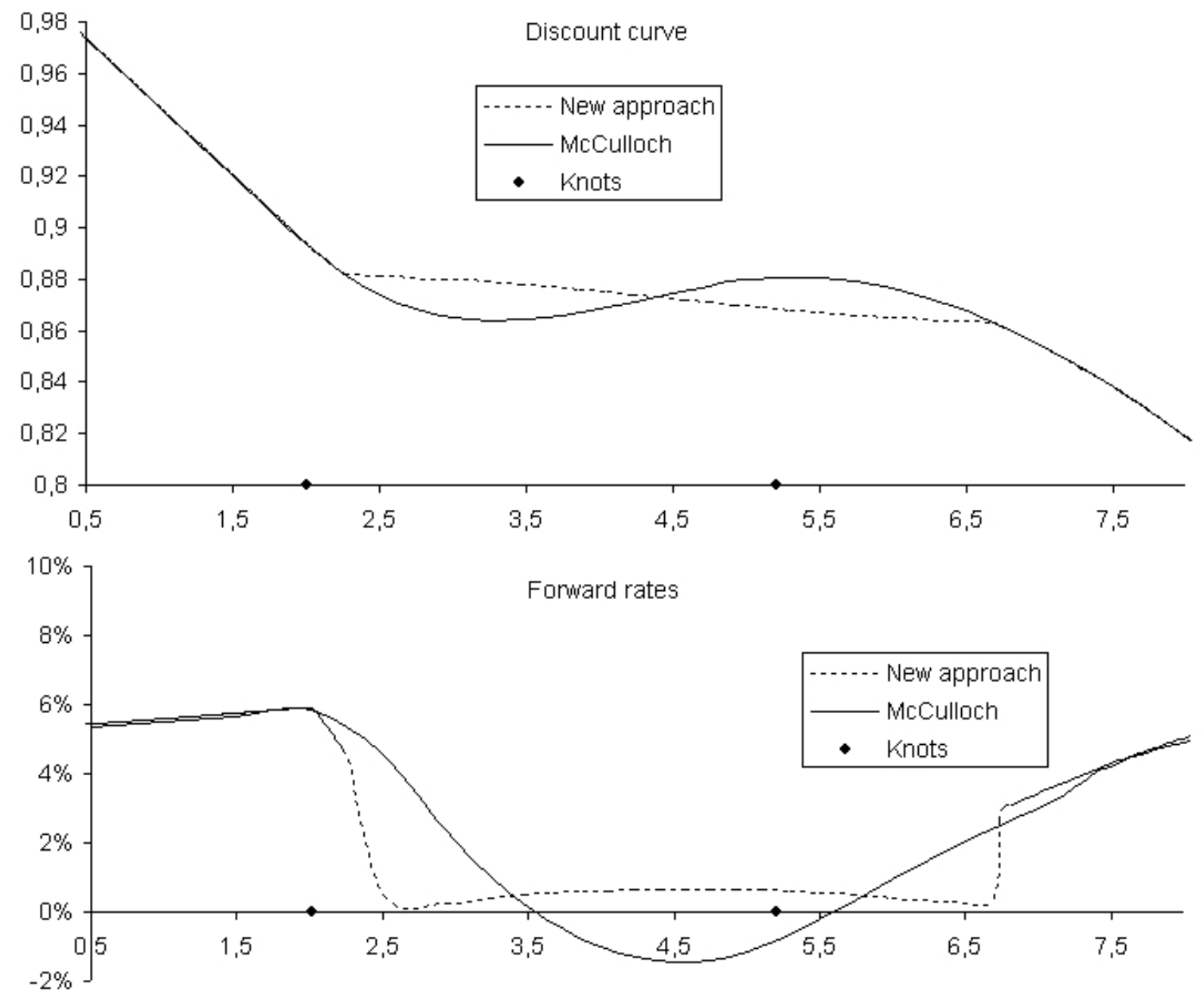

Figure 4: Estimated discount curve and forward rates of Finnish government bonds. 

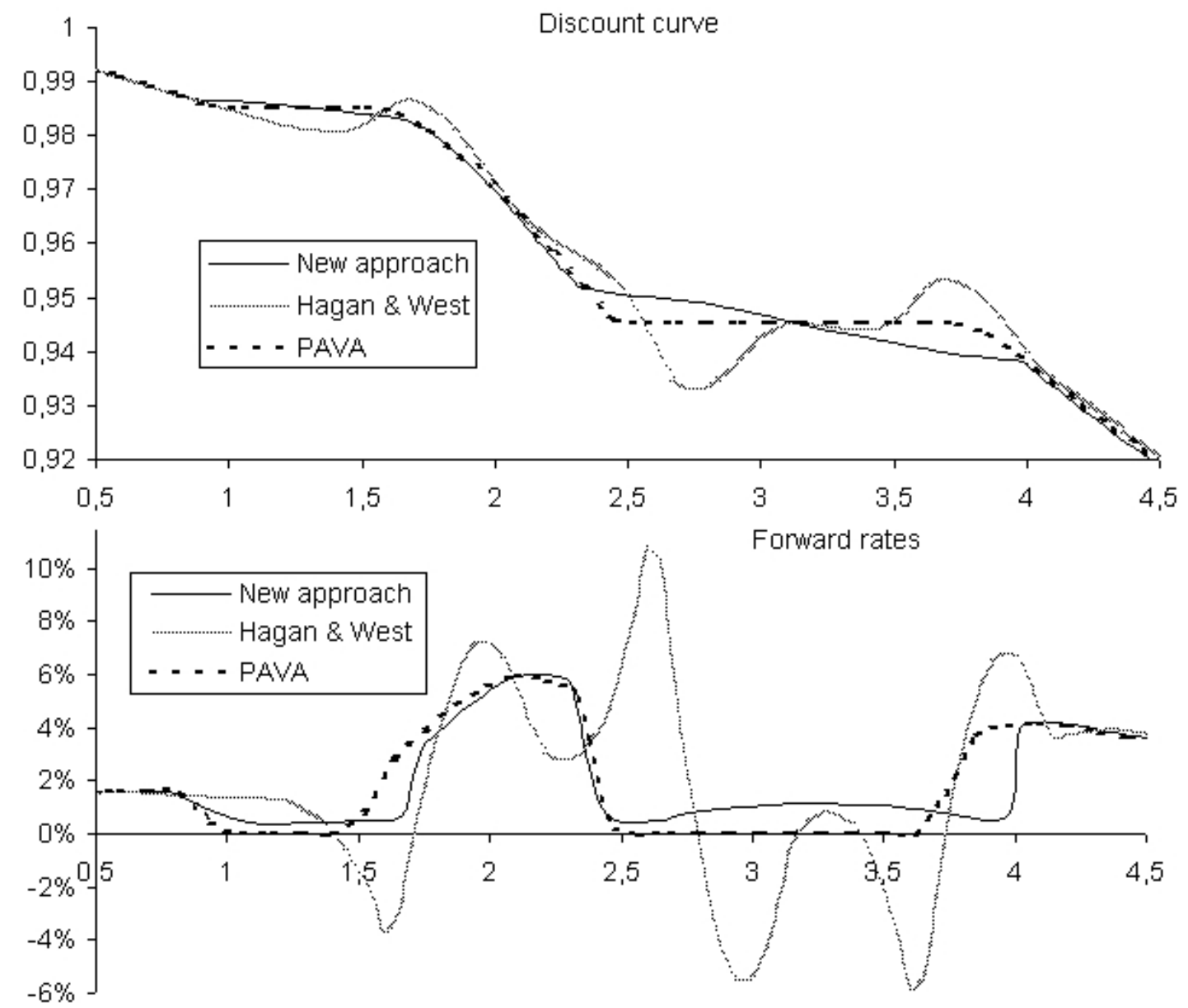

Figure 5: Estimated discount curve and forward rates of Swiss mortgage bonds. 

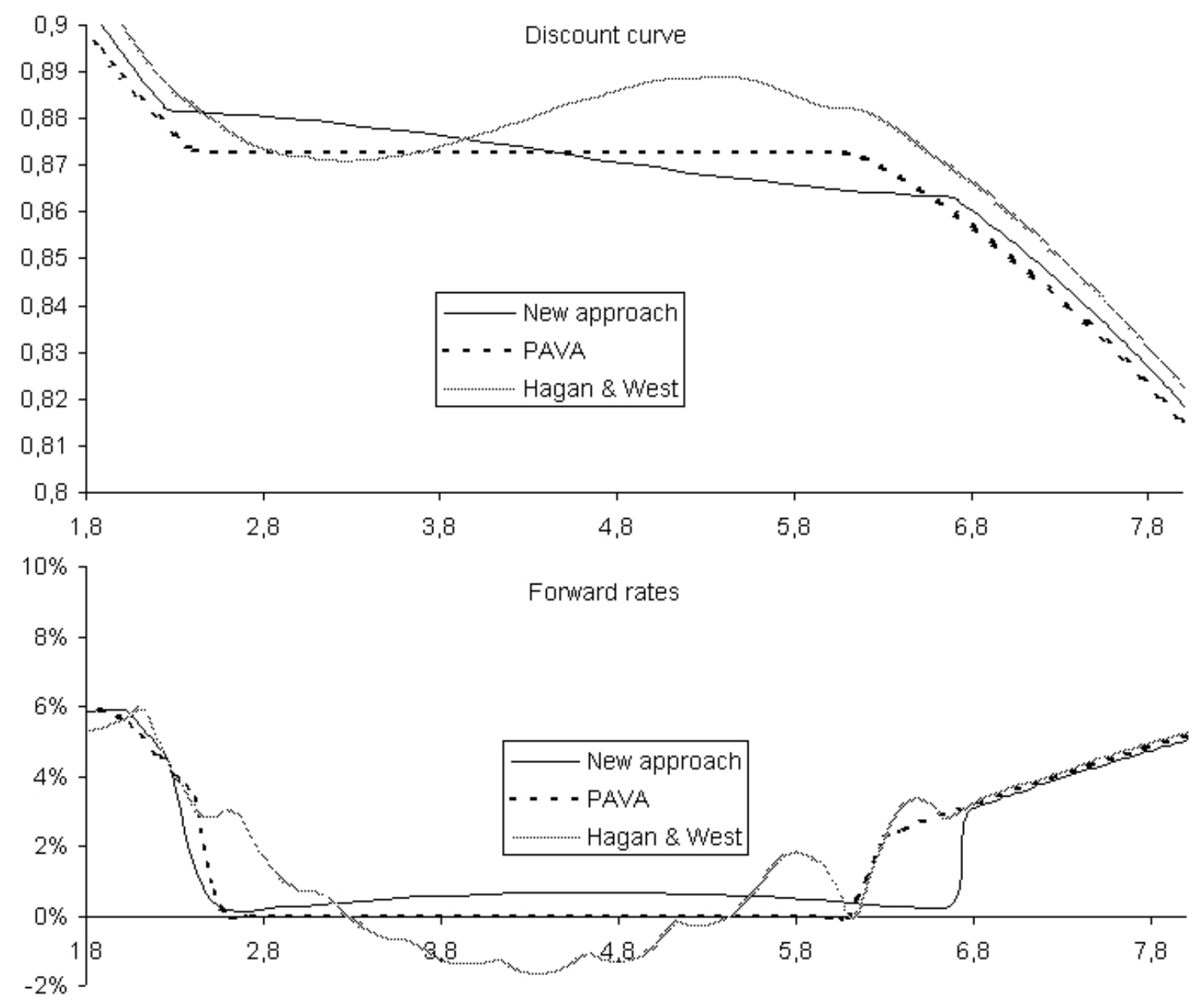

Figure 6: Estimated discount curve and forward rates of Finnish government bonds. 